\title{
On the characterisation of tracer particles for thermographic particle image velocimetry
}

\author{
Benoit Fond · Christopher Abram • Frank Beyrau
}

Received: 30 October 2014 / Accepted: 19 December 2014 / Published online: 9 January 2015

(c) The Author(s) 2015. This article is published with open access at Springerlink.com

\begin{abstract}
Recent studies have shown that thermographic phosphor particles are suitable tracers for simultaneous temperature and velocity imaging in gas flows. For the development of this thermographic particle image velocimetry technique, the choice of phosphor is a key consideration and new phosphor materials need to be identified and their luminescence properties thoroughly characterised. This paper describes a method for investigating phosphor particles for gas thermometry. The method consists of performing spectroscopic experiments on particles dispersed in the gas, while simultaneously measuring the number density of tracer particles using a particle counting system, which is based on high-resolution Mie-scattering images. The measurement of the seeding density allows a direct comparison of different phosphors on a "per particle" basis and estimation of the effect of the particles on the gas thermal properties. The effect of parameters such as the excitation fluence, gas composition and temperature on the luminescence emission intensity can be studied independently of the seeding density. As a demonstration, this system is used to investigate micron-size $\mathrm{BAM}: \mathrm{Eu}^{2+}$ particles. The necessary seeding density for precise temperature measurements is determined, and a nonlinear dependence of the luminescence emission intensity on the excitation fluence is identified.
\end{abstract}

B. Fond

Department of Mechanical Engineering, Imperial College

London, London SW7 2AZ, UK

C. Abram · F. Beyrau ( $\square)$

Lehrstuhl für Technische Thermodynamik, Otto-von-Guericke

Universität Magdeburg, 39106 Magdeburg, Germany

e-mail: frank.beyrau@ovgu.de

\section{Introduction}

Laser-based measurement techniques have greatly improved our fundamental understanding of complex flows involving heat transfer or chemical reactions. In particular, techniques capable of simultaneously measuring scalar and velocity fields provide invaluable physical insight into the interactions between different physical phenomena, e.g. turbulence, chemistry and buoyancy forces.

Recently, a novel technique for simultaneous temperature and velocity imaging has been developed. It is based on thermographic phosphors, which are solid materials with temperature-dependent luminescence properties that can be used for remote thermometry. Micron-sized phosphor particles are seeded into the flow as a tracer. For velocimetry, laser light elastically scattered by the particles is recorded using a conventional particle image velocimetry (PIV) approach. Simultaneously, the same particles are excited by an additional UV laser source, and their luminescence emission is recorded to determine the particle temperature. Typically, the temperature dependence of the luminescence spectrum is exploited by simultaneously imaging the emission in two spectral regions, chosen so that the intensity ratio of the two signals is a function of the temperature. It has been demonstrated that for micronsize particles, the temperature and velocity of the particle match that of the surrounding gas in most turbulent flows of practical interest [1]. This thermographic PIV technique has been used for time-averaged [2] and single-shot [1, 3, 4] measurements of temperature and velocity fields in turbulent flows. Recently, experiments at $\mathrm{kHz}$ repetition rates for time-resolved measurements were demonstrated [5].

The thermographic PIV concept has several advantages. It requires relatively simple instrumentation in terms of lasers and cameras and only a single seeded tracer. In 
contrast to gaseous tracers used for laser-induced fluorescence thermometry, thermographic phosphors are often chemically inert, have a high-melting point $(>2,000 \mathrm{~K})$ and are insensitive to pressure changes and to the gas composition, making them particularly suitable as a tracer for reacting flow applications.

As with all optical flow measurement techniques, the further development of the technique involves increasing the sensitivity, improving the robustness to interference, extending the measurement range (e.g. to temperatures above $1,000 \mathrm{~K}$ ) and simplifying the experimental set-up.

There is an almost unlimited variety of phosphors, with a broad range of luminescence properties and ways in which the emission is influenced by temperature [6]. Thus for a particular application, the choice of phosphor is a key experimental consideration. However, the temperature response of the vast majority of phosphors has not been investigated, let alone for their specific use as temperature tracers in turbulent gas flows.

The intrinsic precision of the technique is determined by two factors: the phosphor sensitivity, i.e. the relative change in intensity ratio with temperature and the signal level. While spectrally resolved measurements can help identify phosphors that have a useful response for thermometry, the knowledge of signal levels is critical when evaluating the suitability of a particular phosphor for flow measurements. The signal level can also depend on parameters such as the excitation wavelength and fluence. Some phosphors may also be prone to, e.g. laser-induced heating, or their luminescence might be dependent on the gas composition. These effects must be investigated, as they affect the accuracy and precision of the measurement in practical applications.

It is obvious that a means to systematically compare signal levels for different phosphors on a "per particle" basis and to investigate factors which affect both signal levels and measured temperatures are essential to further develop their use for gas thermometry.

Until now, most studies on thermographic phosphors were aimed at applications for surface thermometry, see, e.g. Refs. [7-9]. These experiments usually involve probing the phosphor in aggregated powder form, placed in a crucible or deposited as a layer on a substrate. This requires comparatively little experimental effort. High signal levels can be achieved due to the high number density of phosphor particles, the particles are immobile so that in principle the conditions can be easily reproduced, and samples can directly be placed in ovens or pressurised chambers. Unfortunately, the information extracted from such measurement is ambiguous. This situation is particularly well summarised in the Phosphor Handbook [6], a text of reference in the phosphor community: "the optical properties of a particle depend on the state of assemblage of particles; a dilute dispersion of particles has different properties from thick powder aggregates such as those encountered in powder beds or in paints. In the former state, the optical character of individual particles dominates, whereas in the latter, complicated multiple scattering occurs". An identical comparison can be made between a dense stack of phosphor particles and the same particles in an extremely dilute suspension, as when seeded into flows for gas thermometry. For seeded particles, the luminescence emission is proportional to the particle absorption cross-section, but for stacked powder, because of multiple scattering, this is not the case and the signal will have a complex dependence on the particle arrangement as well as the absorption and scattering cross-section of the individual particles. It would also be impossible to determine the emission intensity of a single particle, which, as discussed below, is of particular significance for gas thermometry using phosphors.

Typically, pulsed lasers with high pulse energies are used to achieve appropriate time resolution and increased signal levels. Powder stacks can be subject to laser-induced heating or permanent damage when subjected to repeated laser illumination, as observed in our laboratory. Intense narrow line emission due to amplified spontaneous emission is another phenomenon which can occur in stacked powder [10], but which is very unlikely in dilute suspensions due to the low subtended solid angle between individual particles.

For these reasons, it is most useful to characterise phosphors in the gas phase, where the nature of the laser-particle interaction exactly matches that of the intended use for temperature-imaging experiments. However, seeding solid micrometre-size particles is a rather empirical science, and it is generally not possible to precisely control the rate of entrainment of particles in the gas stream in a reproducible way and over extended periods of time.

Hence measuring the number of particles that are being probed for characterisation is crucial for two reasons. First, fluctuations in the seeding density can be accounted for when varying certain parameters (such as the excitation wavelength or fluence), to investigate the effect on the luminescence signal. This provides repeatable quantification of light levels for a given set of conditions, and a direct comparison of the overall signal levels from different phosphors on a "per particle" basis.

The second reason is of more fundamental importance. With thermographic PIV, the collected light intensity is in principle a linear function of the number of particles probed. As a result, by seeding the fluid with excessively high particle number densities, a considerable signal level can be obtained, even with a phosphor with luminescence properties which are unfavourable for gas thermometry (low absorption coefficient, low quantum efficiency or long lifetime, i.e. in the millisecond range). Excessive seeding 
densities can decrease the spatial resolution of the technique due to multiple scattering. Also, the gas thermal properties can be altered, in particular the volumetric heat capacity. By measuring the seeding density, the emission intensity of single particles can be determined in order to evaluate, for a given phosphor, the range of seeding densities needed for precise thermometry and whether in these conditions, the particles have any effect on the gas properties.

This communication describes such a particle counting system. The technique uses high-resolution Mie-scattering images of seeded particles, instantaneously sampled in the same probe volume as the detection system for the luminescence emission. The number of particles can be related to luminescence signals, and co-dependencies of the intensity on other parameters can also be examined independently of the seeding density. The system is demonstrated by characterising the signal level of $\mathrm{BAM}: \mathrm{Eu}^{2+}$ phosphor particles dispersed in the gas, showing how this relates to the temperature precision, and how the emission intensity depends on the laser fluence.

\section{Experimental set-up}

\subsection{Phosphor particles and particle seeding}

$\mathrm{BAM}: \mathrm{Eu}^{2+}$ thermographic phosphor particles (KEMK63/ UF-P1, Phosphor Technology, median diameter (based on volume): $2.4 \mu \mathrm{m}$ ) were seeded into a jet of air, $40 \mathrm{~mm}$ in diameter using an in-house built reverse cyclone seeder. Prior to use, a $\mathrm{SiO}_{2}$ nanoparticle coating $\left(\operatorname{Ludox}^{\circledR}\right)$ was applied to the phosphor particles to reduce inter-particle forces by cancelling surface charges, and the powder was dried for $8 \mathrm{~h}$ in a furnace at $125^{\circ} \mathrm{C}$. The seeding density was adjusted by controlling the air velocity in the seeder, which was achieved by changing the pressure drop in a stream that bypassed the seeder.

\subsection{Particle counting}

The aim of the particle counting system is to measure the number density of phosphor particles, irrespective of size, shape or orientation. To identify the presence of phosphor particles, the Mie-scattering signal from these particles was collected at $90^{\circ}$ from the laser illumination. In theory, the scattering intensity at this angle does not carry any direct information on the particle size and is influenced in a complex and nonlinear way by the particle shape and orientation. For spherical particles, for example, while the overall scattering cross-section strongly increases with the particle diameter, the collected intensity at $90^{\circ}$ detection angle is only a weak function of the particle diameter between

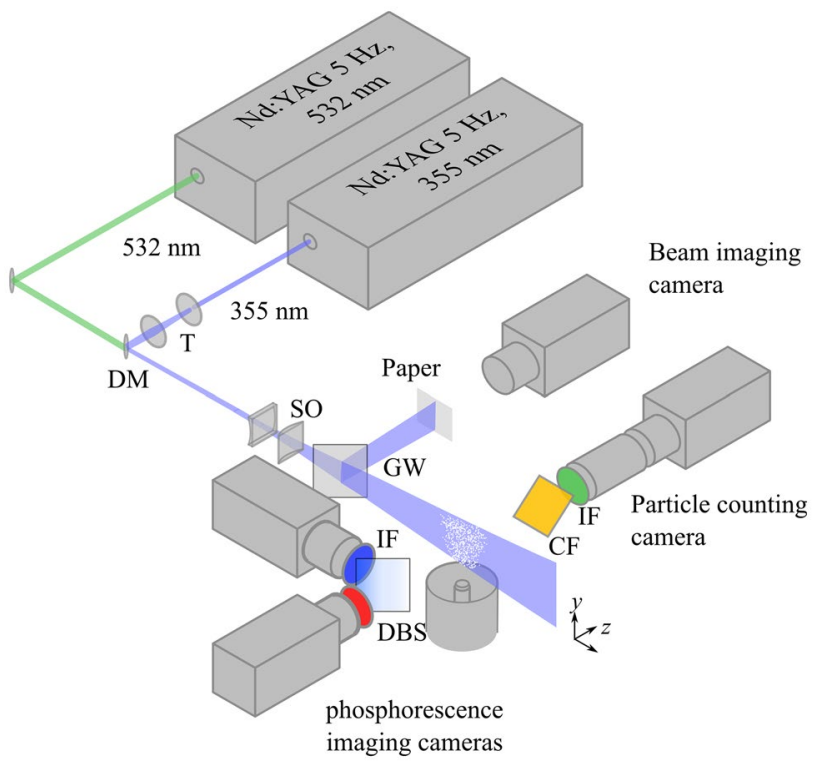

Fig. 1 Experimental set-up. $T$ telescope, $D M$ dichroic mirror, $S O$ sheet optics, $G W$ glass window, $I F$ interference filter, $C F$ coloured glass filter, $D B S$ dichroic beamsplitter

$500 \mathrm{~nm}$ and $3 \mu \mathrm{m}$ [11]. Therefore, using this system, the presence of particles can be detected with equivalent certainty over this range of particle size.

The particles were illuminated with light from a frequency-doubled Nd-YAG laser (Quanta-Ray GCR-150, Spectra-Physics) operating at $5 \mathrm{~Hz}$. Using a cylindrical lens $(+750 \mathrm{~mm})$, a thin light sheet $(160 \mu \mathrm{m})$ was formed in the measurement plane (Fig. 1).

A hardware-binned $(2 \times 2)$ interline transfer CCD camera equipped with a $2 \times$ teleconverter (Tamrom AF $2 \times$ ), a $105-\mathrm{mm} \mathrm{f} / 2.8$ lens (Nikkor) with the f-stop at $\mathrm{f} / 4$, a 532 $10 \mathrm{~nm}$ interference filter (LaVision) and an O.D. 2 neutral density filter was used to record particle images at $90^{\circ}$. A colour-glass filter (OG515, Schott) was positioned in front of the detection system in order to absorb the phosphorescence light directed towards the Mie-scattering camera which would otherwise be reflected by the $532-\mathrm{nm}$ filter and eventually interfere with the phosphorescence detection channels (see below). The filter was positioned at $45^{\circ}$ to reject any specular reflections.

The collection optics provided a magnification of 1.12, and a field of view of $6 \times 8 \mathrm{~mm}$. The FWHM of the image of a single particle was less than a binned camera pixel or $12.9 \mu \mathrm{m}$. This resolution is sufficient to ensure that bias caused by the inability to resolve closely spaced particles is negligible for particle number densities of the order of $10^{11}$ particles $/ \mathrm{m}^{3}$.

The laser pulse energy was adjusted so that the peak intensities of the weakest particle signals were well above the noise threshold. 
Particles images were then processed using Matlab in order to evaluate the number of local maxima in a procedure described below. First, a cut-off filter of 10 counts is applied. The counting algorithm then sorts pixels by descending order of intensities. The position of the maximum is marked, and all pixels within a radius of 2 pixels from this maximum were removed from the list, in order to remove the wings of high peak intensity particle images. In this way, maxima are found until all pixels have been evaluated, and the number of local maxima in each image is used as a measure of the number of particles.

\subsection{Phosphorescence excitation and detection}

A frequency tripled Nd:YAG laser (Quanta-Ray Lab-150, Spectra-Physics) was used for simultaneous phosphorescence excitation. The 532- and 355-nm beams were overlapped in the measurement plane using long-pass dichroic mirrors before the +750 -mm cylindrical lens mentioned above. The UV sheet thickness in the measurement plane (680 $\mu \mathrm{m}$ ) was set independently of the particle counting system by controlling the beam size before the dichroic mirror using a Galilean telescope.

The phosphorescence emission from the particles was detected by two hardware-binned $(4 \times 4)$ non-intensified interline transfer CCD cameras (Imager Intense, LaVision) with $50 \mathrm{~mm}$ f/1.4 Nikon lenses.

Two high-transmission (>80 \%) interference filters at 440-90 and 464-40 $\mathrm{nm}$ and a long-pass dichroic plate beamsplitter (Chroma 445 LP), AR-coated on the reverse side were used to exploit the temperature dependence shift and broadening of the emission line for the ratio-based temperature measurement [1]. The camera exposure time was set to $5 \mu \mathrm{s}$, beginning $1 \mu \mathrm{s}$ before the laser pulse. Since the phosphorescence lifetime of BAM:Eu ${ }^{2+}$ is about a microsecond at room temperature, the whole phosphorescence decay was captured during the exposure. The field of view of the cameras was $28 \times 21 \mathrm{~mm}$.

A $7 \times 7$ moving average filter was applied to the images for a final spatial resolution of $600 \mu \mathrm{m}$.

For measurements of absolute phosphorescence intensity, the recorded intensity in the transmission camera (equipped with the 464-40 nm filter) was averaged over a region that matches the field of view of the counting camera $(8 \times 6 \mathrm{~mm})$.

To evaluate the achievable temperature precision, image pairs were divided to generate the ratio images. In order to correct for non-uniformities in the light collection efficiency, the ratio images were themselves divided by an average ratio image obtained at room temperature. Prior to the experiment, the cameras/beamsplitter system was carefully adjusted using kinematic stages as described in [1]. Using this procedure, the mean residual displacement between the two recorded phosphorescence images was 0.15 pixels, as determined by cross-correlation and no software mapping was applied before division of the images.

After these corrections, the ratio images were converted to temperature using in-flow calibration data obtained previously with the same detection system (camera and spectral filters).

\subsection{Laser energy and sheet profile measurements}

To accurately size the probe volumes in order to determine the seeding density and the excitation fluence, the laser sheet profiles were measured at the start of the experiments. The laser sheets were reflected using a glass window with an AR coating on the reverse side onto a thin piece of paper in a position that matched the windowmeasurement volume distance [12]. This paper was imaged using a CCD camera equipped with an 85-mm Nikon lens. The resolution of the imaging system was checked using a USAF target. The images of the sheets reflected from both the front and the back surface of the glass window were found to have the same FWHM thickness, confirming the homogeneity and linear character of the paper fluorescence. However, to avoid any photo bleaching of the paper after repeated illumination, a preferable method would be to directly illuminate the camera with the attenuated laser beam.

A photodiode-based energy-monitoring unit (Energy monitor V9, LaVision) was used to measure the energies of the lasers on a shot-to-shot basis. This system was precalibrated using a pyroelectric energy detector (PEM-45 K, Radiant Dyes).

\section{Results}

\subsection{Emission intensity per particle}

To quantify the mean phosphorescence intensity of single $2 \mu \mathrm{m}$ BAM: $\mathrm{Eu}^{2+}$ particles, the number of particles present in the probe volume was measured using the particle counting system. The seeding density was varied while phosphorescence and Mie-scattering images were recorded simultaneously and on a shot-to-shot basis. It can be seen in Fig. 2 that the recorded phosphorescence signal is a linear function of the seeding density $\left(R^{2}=94 \%\right)$, indicating also that there is no detectable attenuation of the phosphorescence signal by particles on the optical collection path at these seeding levels.

To determine the necessary seeding density for a given phosphor, single-shot single-pixel statistics were compiled to determine the temperature precision at various seeding levels. 


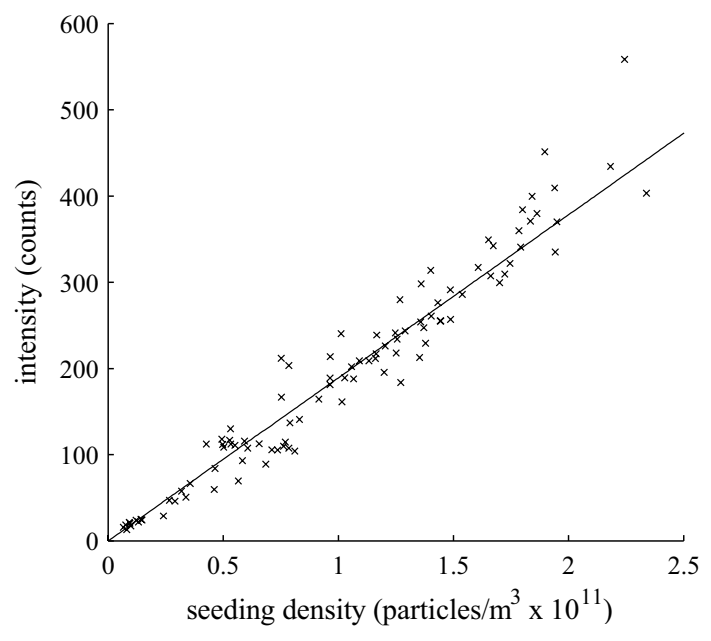

Fig. 2 Recorded pixel intensity in the $464-40 \mathrm{~nm}$ channel for $2-\mu \mathrm{m}$ $\mathrm{BAM}: \mathrm{Eu}^{2+}$ particles as a function of the seeding density for $355-\mathrm{nm}$ excitation with a fluence of $23 \mathrm{~mJ} / \mathrm{cm}^{2}$

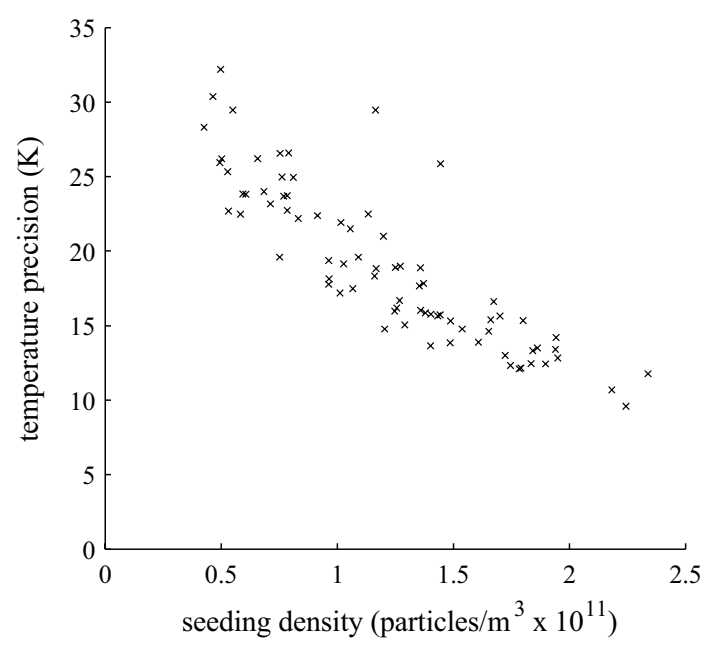

Fig. 3 Temperature precision at room temperature as a function of the seeding density for 355-nm excitation with a fluence of $23 \mathrm{~mJ} /$ $\mathrm{cm}^{2}$

Figure 3 shows the variation of the temperature precision with the seeding density, measured for an excitation fluence of $23 \mathrm{~mJ} / \mathrm{cm}^{2}$. Since the collected phosphorescence intensity is proportional to the seeding density, the signalto-noise ratio also increases with the seeding density and the measurement precision improves. For reference, a $10 \mathrm{~K}$ deviation corresponds to a $2 \%$ change in the normalised ratio.

For PIV measurements, the presence of at least 15 particles on average in each interrogation area is recommended [13] and therefore a minimum seeding density of $10^{11}$ particles $/ \mathrm{m}^{3}$ is needed for a resolution of $600 \mu \mathrm{m}$. With these phosphor particles, the same seeding density generates enough signal to maintain a temperature precision better than $20 \mathrm{~K}$ between 300 and $600 \mathrm{~K}$.

With knowledge of the absolute seeding density the effect of the particles on the bulk gas properties can be determined. For a particle number density of $2 \times 10^{11}$ particles $/ \mathrm{m}^{3}$, the heat capacity of the gas is increased by only $0.2 \%$ at $300 \mathrm{~K}$ and by $0.5 \%$ at $1,100 \mathrm{~K}$. Based on particle-fluid thermal conductivity calculations proposed by Maxwell [14], at this level of seeding, the particles have no effect on the thermal conductivity of the gas due to the very little volume fraction occupied by the particles. These results show that these particles have a negligible effect on the gas thermal properties. Phosphors with a longer lifetime, poor absorption or a lower quantum yield would require considerably higher seeding densities in order to achieve useful signal intensities, increasing the heat capacity of the gas, and possibly quenching chemical reactions.

\subsection{Seeding density correction for quantitative investigation of particle phosphorescence}

Figure 4 shows the raw phosphorescence intensity during a typical recording sequence, at a constant (room) temperature and constant laser pulse energy. Significant fluctuations in the signal are present, mainly due to seeding density fluctuations. Were another parameter, e.g. the laser fluence, varied during this experiment, these fluctuations would obscure the influence of this parameter on the phosphorescence intensity. When dividing by the instantaneous seeding density, the resulting signal level is approximately constant, as expected for these conditions. Using this

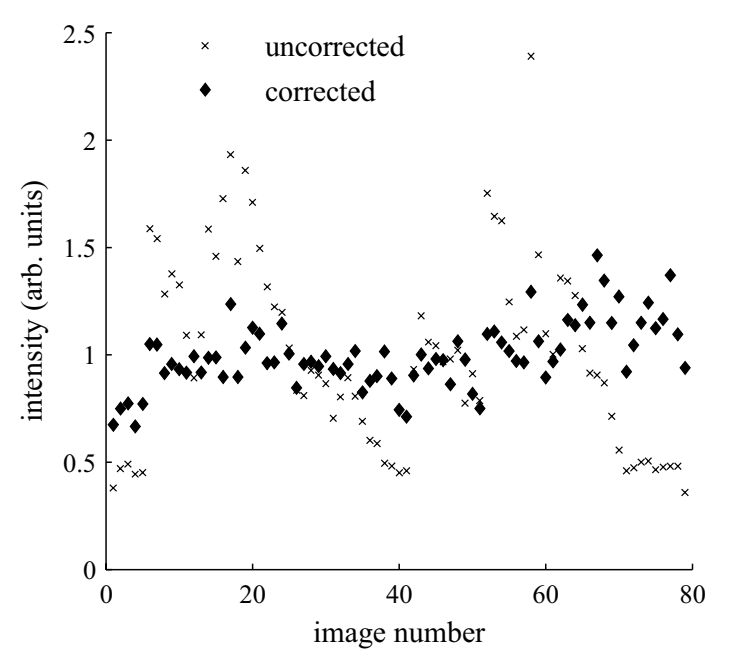

Fig. 4 Evolution of the detected phosphorescence intensity in the 464-40 nm channel at room temperature and at fixed laser fluence, before and after correction for the instantaneous seeding density. The intensity is normalised to the mean value 


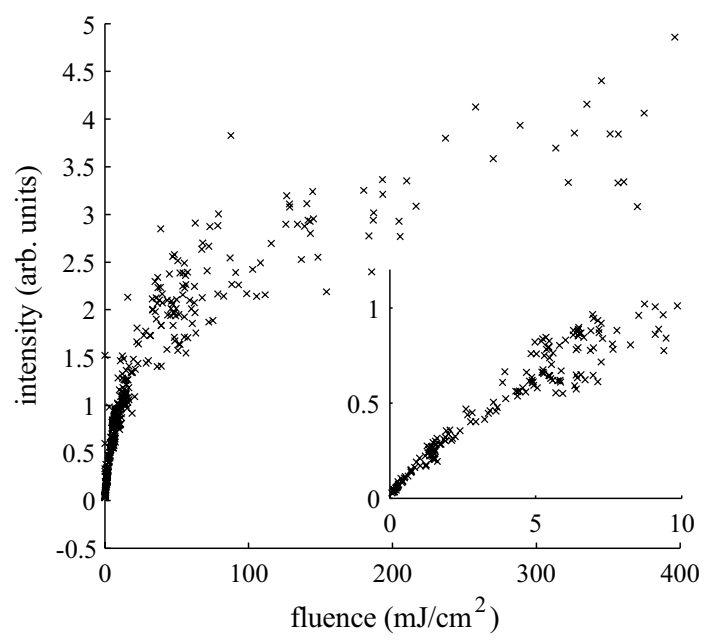

Fig. 5 Phosphorescence intensity in the 464-40 nm channel with increasing laser fluence in the gas phase, corrected for the instantaneous seeding density including a detailed view. The intensity is normalised to the intensity obtained for a fluence of $10 \mathrm{~mJ} / \mathrm{cm}^{2}$

approach, various effects on the particle phosphorescence intensity can now be isolated.

\subsection{Effect of the excitation fluence on the phosphorescence intensity}

As an example to demonstrate the utility of this particle counting approach, the dependence of the phosphorescence intensity on the excitation fluence was investigated.

The laser energy was varied during a recording sequence, and the phosphorescence intensity, seeding density and laser energy were measured simultaneously.

A clear nonlinear dependence of the intensity on the excitation fluence can be observed, as shown in Fig. 5. Below $2-3 \mathrm{~mJ} / \mathrm{cm}^{2}$, the phosphorescence intensity is a rather linear function of the laser fluence. Above this value, the rate of increase gradually drops with laser fluence before reaching again a rather constant value above around $25 \mathrm{~mJ} / \mathrm{cm}^{2}$. At a fluence of $400 \mathrm{~mJ} / \mathrm{cm}^{2}$, the phosphorescence intensity is by an order of magnitude lower than expected from a linear behaviour, giving clear evidence of saturation.

\section{Conclusion}

In this paper, we identify a need for a methodology to statistically characterise the luminescence of dispersed phosphor particles for their use as gas-phase tracers for simultaneous temperature and velocity measurements.

Little information can be extracted from measurements performed on aggregated powder because its optical and thermal properties can be very different from those of individuals particles suspended in a gas. Multiple scattering, heating or damage of the phosphor after repeated illumination and amplified spontaneous emission contribute to the inaccuracy or lack of reproducibility. In addition, it is not possible to determine the emission intensity per particle from powder-based measurements. We conclude that these investigations must be performed in the gas phase.

However, when seeding solid particles, it is not possible to control the rate of entrainment of particles into the stream, which has a strong effect on signal levels. A particle counting system was therefore developed, which uses high-resolution particle images to measure the instantaneous seeding density. It presents several advantages. It uses the same hardware as the actual thermographic PIV system, making it a simple matter to convert existing systems for phosphor characterisation, where effectively the PIV laser and camera are used for particle counting. The probe volume of the counting system is well matched in terms of inplane dimensions to that of the luminescence detection, and the measurements are simultaneous.

The system was used for the investigation of $2-\mu \mathrm{m}$ BAM:Eu particles. The signal level is shown to be proportional to the seeding density. Results also indicate that for this phosphor a seeding density comparable to that used in conventional PIV experiments provides sufficient signal levels for precise thermometry. Calculations show that at this level of seeding the particles have negligible effect on the gas properties.

Using this system, the luminescence intensity can be decoupled from strong temporal fluctuations in the seeding. As an example, the diagnostics were used to clearly identify the sub-linear dependence of the phosphorescence intensity on the laser fluence above $2 \mathrm{~mJ} / \mathrm{cm}^{2}$ for $2-\mu \mathrm{m}$ BAM:Eu particles. The potential increase in signal when using different excitation wavelengths or linewidths, and the effect of temperature, pressure and gas composition can be identified in a similar manner.

There is an almost infinite number of phosphors with different sensitivities and covering different temperature ranges, so a phosphor can be chosen for a specific application. Other phosphors currently used for gas thermometry (e.g. $\mathrm{Mg}_{4} \mathrm{FGeO}_{6}: \mathrm{Mn}^{4+}$ [3], YAG: $\mathrm{Pr}^{3+}$ [4]) must be characterised in a similar fashion to ensure that excessive seeding densities are not required to achieve a reasonable precision and to check cross-dependencies on other parameters. The simple tools described here should also be used for the study of promising phosphors in order to extend the capabilities of this measurement technique.

Acknowledgments The financial support for parts of this work from the UK Engineering and Physical Science Research Council (EPSRC) is gratefully acknowledged. 
Open Access This article is distributed under the terms of the Creative Commons Attribution License which permits any use, distribution, and reproduction in any medium, provided the original author(s) and the source are credited.

\section{References}

1. B. Fond, C. Abram, A.L. Heyes, A.M. Kempf, F. Beyrau, Simultaneous temperature, mixture fraction and velocity imaging in turbulent flows using thermographic phosphor tracer particles. Opt. Express 20, 22118-22133 (2012)

2. A. Omrane, P. Petersson, M. Alden, M.A. Linne, Simultaneous 2D flow velocity and gas temperature measurements using thermographic phosphors. Appl. Phys. B Lasers O 92, 99-102 (2008)

3. S. Someya, Y. Okura, M. Uchida, Y. Sato, K. Okamoto, Combined velocity and temperature imaging of gas flow in an engine cylinder. Opt. Lett. 37, 4964-4966 (2012)

4. N.J. Neal, J. Jordan, D. Rothamer, Simultaneous measurements of in-cylinder temperature and velocity distribution in a smallbore diesel engine using thermographic phosphors. SAE Int. J. Eng. 6, 300-318 (2013)

5. C. Abram, B. Fond, A.L. Heyes, F. Beyrau, High-speed planar thermometry and velocimetry using thermographic phosphor particles. Appl. Phys. B Lasers O 111, 155-160 (2013)

6. S.Y. Shionoya, W.M. Yen, H. Yamamoto, Phosphor Handbook (CRC Press, Boca Raton, 2006)
7. S.W. Allison, G.T. Gillies, Remote thermometry with thermographic phosphors: instrumentation and applications. Rev. Sci. Instrum. 68, 2615-2650 (1997)

8. M. Aldén, A. Omrane, M. Richter, G. Särner, Thermographic phosphors for thermometry: a survey of combustion applications. Prog. Energy Combust. 37, 422-461 (2010)

9. J. Brübach, C. Pflitsch, A. Dreizler, B. Atakan, On surface temperature measurements with thermographic phosphors: a review. Prog. Energy Combust. 39, 37-60 (2013)

10. V.M. Markushev, M.V. Ryzhkov, C.M. Briskina, H. Cao, UV radiation of powdered $\mathrm{ZnO}$ pumped by nanosecond pulses. Laser Phys. 15, 1611-1616 (2005)

11. C.F. Bohren, D.R. Huffman, Absorption and Scattering of Light by Small Particles (Wiley, New York, 1983)

12. S. Pfadler, F. Dinkelacker, F. Beyrau, A. Leipertz, High resolution dual-plane stereo-PIV for validation of subgrid scale models in large-eddy simulations of turbulent premixed flames. Combust. Flame 156, 1552-1564 (2009)

13. R.D. Keane, R.J. Adrian, Optimization of particle image velocimeters. 1. Double pulsed systems. Meas. Sci. Technol. 1, 1202 1215 (1990)

14. J. Maxwell, A Treatise on Electricity and Magnetism (Clarendon Press, Oxford, 1873) 